\title{
Caracterização dos Estratos Verticais de Pastagens Urochloa brizantha cv. Marandu Manejadas em Diferentes Alturas
}

\section{Characterization of the Pastures Vertical Strata of Urockloa brizantha cv. Marandu Managed at Different Heights}

Rafaela Machado dos Santos ; José Victor Pronievks Barreto ${ }^{a}$; Mateus Ludovico Zambotia; Camila Cano Serafim ${ }^{\text {a }}$, Kivia Cardoso Oliveira ${ }^{\mathrm{b}}$; Marilice Zundtc; Júlio César Damasceno ${ }^{\mathrm{d}}$; Cristiano Côrtes ${ }^{\mathrm{e}}$; Marcelo Diniz dos Santos ${ }^{\mathrm{a}}$; Simone

Fernanda Nedel Pertilea; Fabíola Cristine de Almeida Rego*a

aUnopar, Programa de Pós-Graduação Stricto Sensu em Saúde e Produção Animal. PR, Brasil.

bUniversidade de Cuiabá. MT, Brasil.

'Universidade do Oeste Paulista. SP, Brasil.

dUniversidade Estadual de Maringá. PR, Brasil.

eAgrinova, Canadá.

*E-mail: fabiola.cristine@kroton.com.br

\begin{abstract}
Resumo
A estrutura da pastagem é uma característica determinante tanto da dinâmica de crescimento da planta quanto do comportamento ingestivo dos animais. O objetivo do trabalho foi descrever a densidade da forragem e a proporção dos componentes morfológicos da planta, nos estratos verticais de pastagens de capim Marandu, submetido ao pastejo em diferentes alturas. O delineamento utilizado foi o inteiramente casualizado e as análises de variância e de regressão. Durante o experimento, as alturas médias dos seis piquetes utilizados foram de 10, 17, 18, 29, 32 e 50 $\mathrm{cm}$. O material coletado foi separado, em lâmina foliar, colmo verde e material morto, que foram analisados quanto ao teor de matéria seca, proteína bruta e fibra em detergente neutro. Os estratos verticais apresentaram diferentes composições em relação aos componentes morfológicos de acordo com a altura do pasto. Os estratos próximos ao solo apresentaram maior densidade de forragem com valores de 370 a $543 \mathrm{~kg} / \mathrm{ha} . \mathrm{cm}$, maior proporção de colmos verdes (30 a 53\%) e material morto (25 a 52\%) e menor de lâminas foliares ( 10 a $35 \%)$. Independente da altura do pasto, os estratos superiores são mais sensíveis aos efeitos do animal e do ambiente, e apresentaram maiores teores de PB e FDN para lâminas foliares e colmos. Nos estratos acima de $15 \mathrm{~cm}$, os valores de PB e FDN das lâminas foliares verdes variaram entre 10,3 e 13,6\% e 49,3 e 54,2\%, respectivamente. O manejo em diferentes alturas altera as características dos estratos verticais do pasto, principalmente, nos estratos acima de $15 \mathrm{~cm}$.

Palavras-chave: Altura do Pasto. Densidade Forrageira. Colmos Verdes. Material Morto. Lâmina Foliar.
\end{abstract}

\begin{abstract}
The pasture structure is a determining characteristic of both the plant growth dynamics and the animals ingestive behavior. The objective of the work was to describe the density and the proportion of the plant'morphological components, in the pastures vertical strata of Marandu grass, subjected to grazing at different heights. The design used was completely randomized and the analysis of variance and regression. During the experiment, the average heights of six paddocks used were 10,17, 18,29,32 and $50 \mathrm{~cm}$. The collected material was separated into leaf blade, green stem and dead material, which were analyzed as to the dry matter content, crude protein and fiber in neutral detergent. The vertical strata presented different compositions in relation to the morphological components according to the height of the pasture. The near-soil strata showed higher forage density with values from 370 to $543 \mathrm{~kg} / \mathrm{ha} . \mathrm{cm}$, higher proportion of green stems (30 to $53 \%$ ) and dead material (25 to $52 \%)$ and smaller number of leaf blades (10 to 35\%). Regardless of the height of the pasture, the upper strata are more sensitive to the animal and the environment effects, and presented higher levels of $C P$ and NDF for leaf blades and stems. In the strata above $15 \mathrm{~cm}$, the values of $P B$ and NDF of the green leaf blades varied between 10.3 and $13.6 \%$ and 49.3 and $54.2 \%$, respectively. The management of different heights change the characteristics of the pasturevertical strata, mainly in the strata above $15 \mathrm{~cm}$.
\end{abstract}

Keywords: Height of the Pasture. Forage Density. Green Stems. Dead Material. Leaf Laminas.

\section{Introdução}

A estrutura do pasto é a forma de distribuição dos componentes morfológicos na planta, nos sentidos horizontal e vertical do pasto (BAUER et al., 2010). Uma das alternativas para caracterizar esta estrutura é a quantificação da composição morfológica e a densidade volumétrica da pastagem, uma vez que o pasto pode apresentar diversas combinações morfológicas, que influenciam na colheita e consumo deste alimento pelo animal (SANTOS et al., 2010).

Nesse sentido, uma pesquisa realizada por Silva et al. (2012) apresentou parâmetros que determinam padrões de comportamento ingestivo dos animais. Os autores verificaram, também, que variações na estrutura do dossel forrageiro podem afetar a ingestão de forragem pelos animais, indicando que podem ser traçadas estratégias de pastejo de acordo com a época do ano e exigências nutricionais.

$\mathrm{O}$ uso de ferramentas de manejo para controlar as alterações na estrutura do pasto ao longo das estações do ano, vem sendo constantemente foco de estudos, visando adequar o consumo do animal em cada estação (SANTOS et al., 2015). Além da variação nos componentes morfológicos, pode haver uma variação entre os estratos em relação às características químicas dos componentes (SILVA et al., 2012), indicando que o valor nutritivo da forragem é alterado em função da 
camada, que está sendo pastejada.

Este estudo investiga a hipótese de que a distribuição vertical dos componentes morfológicos e a composição química variam em função da altura do pasto. Com isso, o objetivo foi descrever o perfil vertical de pastos de Urochloa brizantha cv. Marandu, quantificando a proporção, a densidade dos componentes morfológicos e a composição bromatológica nos estratos verticais da planta, manejada em diferentes alturas, sob pastejo. Objetivou-se, ainda, gerar equações que permitam descrever os horizontes verticais dos pastos de capim Marandu em função da altura do pasto para auxílio na construção de modelos de predição de ingestão animal para esta espécie.

\section{Material e Métodos}

O experimento foi realizado na Fazenda Experimental de Iguatemi (FEI), da Universidade Estadual de Maringá, localizada no distrito de Iguatemi, na região Noroeste do Paraná, sob as coordenadas geográficas $23^{\circ} 22^{\prime}$ ' 12' latitude Sul e 52 03 " 54' longitude Oeste. O solo da região é classificado como latossolo vermelho distrófico (SIBCS, 2013). O clima predominante na região é o Cfa subtropical úmido mesotérmico (IWAMOTO et al., 2015).

A forrageira avaliada foi o capim Urochloa brizantha cv. Marandu manejadas em diferentes alturas. No plantio foi realizada adubação com $800 \mathrm{~kg} / \mathrm{ha}$ (0-10-10 NPK). A aplicação de $\mathrm{N}$ foi feita a lanço, na forma de ureia, dividida em 3 aplicações (70, 70 e $60 \mathrm{~kg}$ N/ha). A área experimental consistiu de $1340 \mathrm{~m}^{2}$ divididos em 6 piquetes de 23,5 por 9,5 $\mathrm{m}$, separados por uma cerca elétrica de um fio. Os piquetes foram mantidos em diferentes alturas por meio do uso de animais em pastejo (vacas e novilhas da raça Holandesa).

No período pré-experimental (60 dias antes das avaliações), as parcelas foram pastejadas pelos animais reguladores com intuito de obter alturas contrastantes nos seis piquetes. Nesse período, a altura das plantas foi mensurada três vezes por semana, com uso de régua, considerando a altura média das folhas da touceira.

Durante período experimental (outubro a fevereiro), as alturas foram mensuradas (Quadro 1) utilizando uma estrutura de $1,2 \mathrm{~m}$ de altura e $60 \mathrm{~cm}$ de comprimento, composto por dois pés de madeira e uma régua de $60 \mathrm{~cm}$ no topo. Nessa régua foram fixados barbantes com ponteiras de metal a cada $5 \mathrm{~cm}$, totalizando 13 pontos para medição. As ponteiras foram deslocadas no sentido vertical, tocando a pastagem, definindo o local para aferir a altura, que foi realizada do topo da planta até o solo. Os piquetes foram regulados em diferentes alturas, previamente ao experimento, através do uso de animais em pastejo. As alturas pretendidas respeitaram as amplitudes normalmente utilizadas no manejo das espécies, como segue: $10,20,30,40,50$ e $60 \mathrm{~cm}$. As alturas médias obtidas foram de $10,17,18,29,32$ e $50 \mathrm{~cm}$. Para atingir a altura de $50 \mathrm{~cm}$, a parcela permaneceu sem animais até o início do experimento. Para obter as médias, a estrutura foi alocada ao acaso em 10 pontos, totalizando 130 observações por piquete por dia.

Quadro 1 - Altura média (cm) diária do pasto e desvio padrão durante os períodos de coletas no capim Marandu

\begin{tabular}{|c|c|c|c|c|c|c|}
\hline \multicolumn{3}{|c|}{ DATAS } & \multicolumn{4}{|c|}{ Parcelas Capim Marandu } \\
\hline & 1 & 2 & 3 & 4 & 5 & 6 \\
\hline $03 / 12$ & $11,0(4,9)$ & $19,8(11,9)$ & $19,7(5,4)$ & $41,0(9,9)$ & $36,1(10,0)$ & \\
\hline $04 / 12$ & $12,0(5,2)$ & $15,7(7,5)$ & $18,0(5,9)$ & $28,3(9,4)$ & $34,6(10,6)$ & \\
\hline $05 / 12$ & $11,0(6,0)$ & $18,9(8,6)$ & $20,5(6,8)$ & $29,3(10,5)$ & $28,0(8,9)$ & \\
\hline $06 / 12$ & $8,0(4,5)$ & $19,3(7,5)$ & $15,7(6,1)$ & $21,0(7,1)$ & $34,7(8,3)$ & \\
\hline $07 / 12$ & $9,0(4,4)$ & $11,2(4,0)$ & $16,3(5,8)$ & $24,8(7,1)$ & $29,1(10,2)$ & \\
\hline$* 18 / 12$ & - & $16,9(7,5)$ & - & - & - & $43,0(13,0)$ \\
\hline$* 19 / 12$ & - & $16,5(7,0)$ & - & - & - & $58,0(17,0)$ \\
\hline Média & 10,2 & 16,9 & 18,12 & 28,9 & 32,52 & 50,5 \\
\hline
\end{tabular}

* Nestas datas foram avaliadas apenas as parcelas 2 e 6.

Fonte: dados da pesquisa.

A densidade da forragem foi estimada através da colheita diária de quatro amostras por piquete, utilizando-se uma estrutura de ferro, de $0,3 \mathrm{~m}$ por $0,3 \mathrm{~m}$, estratificados a cada 15 $\mathrm{cm}$ a partir do solo (estrato 1: $0-15 \mathrm{~cm}$, estrato 2: $15-30 \mathrm{~cm}$, estrato 3: $30-45 \mathrm{~cm}$, estrato $4: 45-60 \mathrm{~cm}$, estrato 5: $60-75$ $\mathrm{cm})$. As estimativas foram baseadas na técnica descrita por Houlderbaun e Sollenberg (1992), com algumas modificações. A forragem colhida por estrato foi separada em lâmina foliar verde, colmo verde (colmo + bainha) e material morto.

Após a separação, foi realizada a pré-secagem dos componentes em estufa de circulação forçada de ar a $55^{\circ} \mathrm{C}$, durante 72 horas. Após esse processo, foi realizada a pesagem e a moagem dos componentes das plantas com peneira de 1 $\mathrm{mm}$ para posteriores análises.

Para cada estrato e seus componentes morfológicos foram realizadas análises para determinação de matéria seca e de proteína bruta (AOAC, 1990); e de fibra em detergente neutro, de acordo com Van Soest et al. (1991).

A partir da colheita estratificada da planta, foram estimadas as seguintes variáveis: proporção de lâminas foliares verdes (LF), proporção de colmos verdes (CV), proporção de material morto (MM), massa de forragem (MF), massa de lâminas foliares verdes (MLV), massa de colmos verdes (MCV) e massa de material morto (MMM) por estrato. A densidade dos componentes morfológicos para cada estrato foi determinada através da razão entre a massa de forragem e a altura do estrato $(15 \mathrm{~cm})$, sendo expressa em $\mathrm{kg} / \mathrm{ha}$. Foram estimadas densidade de lâminas foliares verdes (DEL), densidade de colmos verdes (DEC) e densidade de material morto (DEM). As densidades foram avaliadas, também, em função da altura 
do pasto, sem comparações entre os diferentes estratos.

Além da descrição dos componentes morfológicos, foi feita a relação entre os componentes participantes de cada estrato e altura da pastagem, através de análise de variância e regressão. O delineamento utilizado foi o inteiramente casualizado.

$\mathrm{Y}_{\mathrm{ijj}}=\beta_{0}+\beta_{1} \mathrm{X}_{1}+\beta_{2} \mathrm{X}_{2}+\beta_{1} \mathrm{X}_{1} / \beta_{2} \mathrm{X}_{2}+\beta_{1} \mathrm{X}_{1} * \beta_{2} \mathrm{X}_{2}+\mathrm{E}_{\mathrm{ijk}}$

Em que:

$\mathrm{Y}_{\mathrm{ijk}}$ : é o valor observado da variável estudada no indivíduo j recebendo o tratamento $i$.

$\beta_{0}$ : Constante geral.

$\beta_{1} X_{1}$ : efeito do estrato estudado ( $\left.\mathrm{i}=1,2,3,4,5\right)$.

$\beta_{2} X_{2}$ : efeito da altura estudada $(j=1,2,3,4,5,6)$.

$\beta_{1} X_{1} / \beta_{2} X_{2}$ : efeito da altura $\mathrm{j}$ dentro do estrato $i$.

$\beta_{1} X_{1}^{*} \beta_{2} X_{2}$ : efeito da interação entre o estrato i e altura $j$.

$\mathrm{E}_{\mathrm{ijk}}$ : erro aleatório associado a cada observação $\mathrm{Y}_{\mathrm{ij \textrm {k }}}$.

As equações geradas permitiram simular a composição dos horizontes verticais dos pastos de capim Marandu em função da altura do pasto. As avaliações realizadas serviram para caracterizar o perfil vertical da planta e a disposição dos componentes para auxiliar no entendimento do comportamento ingestivo do animal a pasto.

\section{Resultados e Discussão}

As características morfológicas dos estratos 1 e 2, aqueles mais rentes ao solo, não apresentaram diferenças da massa de forragem ( $\mathrm{kg} / \mathrm{ha}$ de MS) entre as alturas estudadas $(\mathrm{p}>0,01)$. Como esperado, a porção mais próxima ao solo, a planta tem elevada produção de MS, que é decorrente da alta proporção de colmos e material morto, independente do manejo adotado. No estrato 1, a proporção de lâminas verdes apresentou comportamento quadrático em função da altura do pasto ( $\mathrm{p}<0,01$ ), aumentando até o ponto de máximo de $29,8 \mathrm{~cm}$ de altura, reduzindo a partir de então (Quadro 2).

Quadro 2 - Equações de regressão e de coeficientes de determinação $\left(\mathrm{R}^{2}\right)$ das variáveis do pasto, nos estratos verticais 1 e 2 , do capim Marandu, em função da altura do pasto

\begin{tabular}{|c|c|c|c|c|}
\hline Variáveis do & Estrato $1(0-15 \mathrm{~cm})$ & & Estrato $2(15-30 \mathrm{~cm})$ & \\
\hline Pasto & Equação de regressão & $\mathrm{R}^{2}$ & Equação de regressão & $\mathrm{R}^{2}$ \\
\hline MF (kg/ha de MS) & $6,07 *$ & & $4,01 *$ & \\
\hline LV $(\%)$ & $Y=-5,81+2,39 X 0,04 X^{2}$ & 0,45 & $Y=-13,57+4,32 X-0,0708 X^{2}$ & 0,44 \\
\hline $\mathrm{CV}(\%)$ & $Y=35,85+0,3325 X$ & 0,15 & $Y=34,45+0,5767 X$ & 0,11 \\
\hline MM (\%) & $Y=76,97-3,33 X+0,051 X^{2}$ & 0,37 & $5,16^{*}$ & \\
\hline PBL (\%) & $10,74 *$ & & $Y=2,27+0,72 \mathrm{~A}-0,01 \mathrm{X}^{2}$ & 0,22 \\
\hline PBC (\%) & $6,33 *$ & & $\mathrm{Y}=1,95+0,45 \mathrm{X}-0,0079 \mathrm{X}^{2}$ & 0,32 \\
\hline PBM (\%) & $\mathrm{Y}=7,07-0,042 \mathrm{X}$ & 0,15 & $\mathrm{Y}=6,72-0,38 \mathrm{X}+0,006 \mathrm{X}^{2}$ & 0,13 \\
\hline FDNL (\%) & $52,83 *$ & & $\mathrm{Y}=27,45+1,59 \mathrm{X}-0,023 \mathrm{X}^{2}$ & 0,17 \\
\hline FDNC (\%) & $64,33 *$ & & $61,88^{*}$ & \\
\hline FDNM (\%) & $63,23 *$ & & $61,13 *$ & \\
\hline
\end{tabular}

MF (massa de forragem), LV (proporção de lâminas foliares verdes), CV (proporção de colmos verdes), MM (proporção de material morto), PBL (proteína bruta das lâminas foliares verdes), PBC (proteína bruta dos colmos verdes), PBM (proteína bruta do material morto), FDNL (fibra em detergente neutro das lâminas foliares verdes), FDNC (fibra em detergente neutro dos colmos verdes), FDNM (fibra em detergente neutro do material morto), * (média), p-valor >0,05. Fonte: Dados da pesquisa.

No pasto com $10 \mathrm{~cm}$, o estrato inferior é acessível aos animais, com maior facilidade de apreensão de folhas, resultando em menor proporção deste item. Uma maior frequência e intensidade de pastejo ocorrida nesta camada, em pastos baixos, pode explicar esse fato, conforme visto também por Gonçalves (2002), pesquisando alturas de 10, 20, 30 e 40 cm também em B. Brizantha.

No estrato 1, a proporção de colmos verdes aumentou linearmente com a altura do pasto $(\mathrm{p}<0,01)$ (Quadro 2), variando de 30 a $53 \%$ nos pastos de 10 a $50 \mathrm{~cm}$ de altura, respectivamente (Quadro 3). O pasto manejado em $10 \mathrm{~cm}$ apresentou uma composição de 52\% de material morto, 30\% de colmos verdes e $18 \%$ de lâminas verdes. Esse padrão de composição morfológica pode limitar a profundidade do bocado do animal, comprometendo a seletividade e o consumo (PAULA et al., 2012).

Quadro 3 - Equação de regressão e coeficientes de determinação $\left(\mathrm{R}^{2}\right)$ das variáveis do pasto, nos estratos verticais 3 e 4 , do capim Marandu em função de diferentes alturas de pastejo

\begin{tabular}{|l|c|c|c|c|}
\hline \multicolumn{1}{|c|}{ Variáveis do } & \multicolumn{2}{c|}{ Estrato 3 (30-45 cm) } & \multicolumn{2}{c|}{ Estrato 4 (45-60 cm) } \\
\hline \multicolumn{1}{|c|}{ Pasto } & Equação de regressão & $\mathbf{R}^{2}$ & Equação de regressão & $\mathrm{R}^{2}$ \\
\hline MF (kg/ha.cm de MS) & $\mathrm{Y}=-1,17+0,09^{\mathrm{a}}$ & 0,72 & $\mathrm{Y}=1,127-0,12 \mathrm{X}+0,003 \mathrm{X}^{2}$ & 0,95 \\
\hline LV $(\%)$ & $\mathrm{Y}=-75,55+9,49 \mathrm{X}-0,14 \mathrm{X}^{2}$ & 0,47 & $\mathrm{Y}=-20,25+1,40 \mathrm{X}$ & 0,27 \\
\hline CV $(\%)$ & $\mathrm{Y}=-7,98+1,17^{\mathrm{a}}$ & 0,39 & $\mathrm{Y}=24,67-2,72 \mathrm{X}+0,06 \mathrm{X}^{2}$ & 0,95 \\
\hline PBL $(\%)$ & $\mathrm{Y}=-11,11+1,55 \mathrm{X}-0,02 \mathrm{X}^{2}$ & 0,53 & $\mathrm{Y}=-3,85+0,24 \mathrm{X}$ & 0,38 \\
\hline PBC $(\%)$ & $\mathrm{Y}=-5,78+0,76 \mathrm{X}-0,011 \mathrm{X}^{2}$ & 0,28 & $\mathrm{Y}=2,94-0,32 \mathrm{X}+0,007 \mathrm{X}^{2}$ & 0,87 \\
\hline FDNL $(\%)$ & $\mathrm{Y}=-34,36+4,80 \mathrm{X}-0,06 \mathrm{X}^{2}$ & 0,51 & $\mathrm{Y}=-19,88+1,21 \mathrm{X}$ & 0,50 \\
\hline FDNC $(\%)$ & $\mathrm{Y}=+0,23+1,38^{\mathrm{a}}$ & 0,25 & $\mathrm{Y}=-20,61+1,101 \mathrm{X}$ & 0,53 \\
\hline
\end{tabular}

MF (massa de forragem), LV (proporção de lâminas foliares verdes), CV (proporção de colmos verdes), PBL (proteína bruta das lâminas foliares verdes), PBC (proteína bruta dos colmos verdes), FDNL (fibra em detergente neutro das lâminas foliares verdes), FDNC (fibra em detergente neutro dos colmos verdes), $\mathrm{p}$-valor $>0,05$.

Fonte: Dados da pesquisa. 
A proporção de material morto apresentou efeito quadrático decrescente $(\mathrm{p}<0,01)$, diminuindo até a altura de 32 $\mathrm{cm}$ e aumentando no pasto de $50 \mathrm{~cm}$ (Quadro 2). A proporção de MM teve comportamento quadrático em função da altura no estrato 1 (Quadro 2), o que não era esperado. Com o aumento da altura do pasto, há um aumento de sombreamento, o que dificulta a entrada de luz na camada inferior do pasto e favorece o acúmulo de folhas senescentes nesta camada. No presente trabalho, para a formação de piquetes com alturas menores $(20 \mathrm{~cm})$, foi necessário um pastejo mais intenso, com maior pisoteio e frequência de desfolha, resultando em maior presença de material morto.

No estrato 2, houve resposta quadrática crescente para proporção de lâminas verdes e linear crescente para a proporção de colmos verdes (Quadro 2). Isso mostra que pastos mais altos apresentam maior concentração de colmos verdes nessa camada. A presença de material morto foi marcante nos estratos 1 e 2, e praticamente inexistente nos demais estratos, por isso não foram analisados.

No estrato 3 , houve aumento linear em relação à altura do pasto para a massa de forragem e proporção de colmos verdes, enquanto a proporção de lâminas verdes apresentou comportamento quadrático crescente (Quadro 3).

No estrato 3 (Quadro 3), a proporção de lâminas verdes aumentou até atingir o máximo de folhas na altura de $33,8 \mathrm{~cm}$ reduzindo a partir de então.

As características químicas das lâminas foliares e colmos verdes sofreram variações ao longo do perfil vertical da planta, e também apresentaram comportamentos diferenciados em função da altura do pasto (Quadros 2 e 4).

Quadro 4 - Média e desvio padrão das variáveis do pasto, nos estratos verticais do capim Marandu, em função de diferentes alturas de pastejo

\begin{tabular}{|c|c|c|c|c|c|c|c|c|c|}
\hline Alturas & Estratos & $\mathrm{MF}$ & DF & LV & $\mathrm{CV}$ & MM & MLV & $\mathrm{MCV}$ & MMM \\
\hline$(\mathrm{cm})$ & $(\mathrm{cm})$ & $\begin{array}{c}\mathrm{Kg} / \mathrm{ha} \mathrm{de} \\
\text { MS }\end{array}$ & $\mathrm{Kg} / \mathrm{ha}$ & $\%$ & $\%$ & $\%$ & $\begin{array}{c}\mathrm{Kg} / \mathrm{ha} \mathrm{de} \\
\text { MS }\end{array}$ & $\begin{array}{c}\mathrm{Kg} / \mathrm{ha} \mathrm{de} \\
\text { MS }\end{array}$ & $\begin{array}{c}\mathrm{Kg} / \mathrm{ha} \mathrm{de} \\
\text { MS }\end{array}$ \\
\hline \multirow[t]{2}{*}{10} & $0-15$ & 5,55 & 370,00 & 18,00 & 30,00 & 52,00 & 63,82 & 122,77 & 183,46 \\
\hline & & $(1,71)$ & $(114,08)$ & $(7,62)$ & $(10,99)$ & $(17,02)$ & $(0,45)$ & $(0,96)$ & $(1,08)$ \\
\hline \multirow[t]{2}{*}{10} & $15-30$ & 1,30 & 87,00 & 36,00 & 58,00 & 6,00 & 31,32 & 50,46 & 5,22 \\
\hline & & $(0,53)$ & $(35,39)$ & $(1,93)$ & $(8,17)$ & $(5,29)$ & $(0,19)$ & $(0,34)$ & $(0,08)$ \\
\hline \multirow[t]{2}{*}{17} & $0-15$ & 6,97 & 464,00 & 19,00 & 48,00 & 33,00 & 93,11 & 219,92 & 151,72 \\
\hline & & $(2,18)$ & $(145,66)$ & $(6,00)$ & $(6,61)$ & $(5,26)$ & $(0,76)$ & $(1,07)$ & $(0,58)$ \\
\hline \multirow[t]{2}{*}{17} & $15-30$ & 2,87 & 191,00 & 37,00 & 58,00 & 5,00 & 70,67 & 110,78 & 9,55 \\
\hline & & $(1,26)$ & $(83,83)$ & $(6,84)$ & $(8,93)$ & $(1,72)$ & $(0,45)$ & $(0,84)$ & $(0,06)$ \\
\hline \multirow[t]{2}{*}{17} & $30-45$ & 0,66 & 44,00 & 60,00 & 40,00 & 0,00 & 26,40 & 17,60 & 0,00 \\
\hline & & $(0,46)$ & $(30,88)$ & $(8,42)$ & $(8,42)$ & $(0,00)$ & $(0,24)$ & $(0,21)$ & $(0,00)$ \\
\hline \multirow[t]{2}{*}{18} & $0-15$ & 8,14 & 543,00 & 23,00 & 46,00 & 31,00 & 124,89 & 249,78 & 168,33 \\
\hline & & $(2,35)$ & $(156,85)$ & $(1,10)$ & $(8,42)$ & $(8,72)$ & $(0,49)$ & $(1,23)$ & $(0,89)$ \\
\hline \multirow[t]{2}{*}{18} & $15-30$ & 2,54 & 169,00 & 53,00 & 39,00 & 8,00 & 89,57 & 65,91 & 13,52 \\
\hline & & $(0,66)$ & $(44,13)$ & $(8,410$ & $(9,35)$ & $(13,66)$ & $(0,35)$ & $(0,36)$ & $(0,42)$ \\
\hline \multirow[t]{2}{*}{29} & $0-15$ & 5,87 & 391,00 & 28,00 & 49,00 & 23,00 & 109,48 & 191,59 & 89,93 \\
\hline & & $(2,07)$ & $(137,69)$ & $(6,82)$ & $(3,23)$ & $(4,35)$ & $(0,42)$ & $(1,05)$ & $(0,75)$ \\
\hline \multirow[t]{2}{*}{29} & $15-30$ & 8,88 & 592,00 & 48,00 & 50,00 & 2,00 & 284,16 & 296,00 & 11,84 \\
\hline & & $(1,31)$ & $(87,17)$ & $(4,23)$ & $(5,20)$ & $(0,00)$ & $(0,67)$ & $(0,66)$ & $(0,02)$ \\
\hline \multirow[t]{2}{*}{29} & $30-45$ & 1,49 & 99,00 & 76,00 & 23,00 & 1,00 & 75,24 & 22,77 & 0,99 \\
\hline & & $(0,78)$ & $(52,14)$ & $(6,43)$ & $(6,43)$ & $(0,00)$ & $(0,59)$ & $(0,21)$ & $(0,00)$ \\
\hline \multirow[t]{2}{*}{32} & $0-15$ & 5,35 & 357,00 & 35,00 & 40,00 & 25,00 & 124,95 & 142,80 & 89,25 \\
\hline & & $(1,55)$ & $(103,66)$ & $(7,35)$ & $(2,33)$ & $(6,43)$ & $(0,59)$ & $(0,68)$ & $(0,54)$ \\
\hline \multirow[t]{2}{*}{32} & $15-30$ & 3,33 & 222,00 & 55,00 & 45,00 & 5,00 & 122,10 & 99,90 & 11,10 \\
\hline & & $(0,89)$ & $(59,53)$ & $(11,7)$ & $(11,7)$ & $(0,00)$ & $(0,70)$ & $(0,47)$ & $(0,00)$ \\
\hline \multirow[t]{2}{*}{32} & $30-45$ & 1,20 & 80,00 & 81,00 & 19,00 & 0,00 & 64,80 & 15,20 & 0,00 \\
\hline & & $(0,68)$ & $(45,37)$ & $(13,1)$ & $(13,1)$ & $(0,00)$ & $(0,41)$ & $(0,32)$ & $(0,00)$ \\
\hline \multirow[t]{2}{*}{32} & $45-60$ & 0,25 & 17,00 & 100,00 & 0,00 & 0,00 & 17,00 & 0,00 & 0,00 \\
\hline & & $(0,25)$ & $(16,65)$ & $(0,00)$ & $(0,00)$ & $(0,00)$ & $(0,25)$ & $(0,00)$ & $(0,00)$ \\
\hline \multirow[t]{2}{*}{50} & $0-15$ & 4,54 & 303,00 & 10,00 & 53,00 & 37,00 & 30,30 & 160,59 & 112,11 \\
\hline & & $(0,65)$ & $(43,47)$ & $(0,12)$ & $(0,32)$ & $(0,44)$ & $(0,07)$ & $(0,36)$ & $(0,22)$ \\
\hline \multirow[t]{2}{*}{50} & $15-30$ & 5,16 & 344,00 & 26,00 & 69,00 & 5,00 & 89,44 & 237,36 & 17,20 \\
\hline & & $(0,78)$ & $(51,88)$ & $(3,26)$ & $(6,78)$ & $(3,51)$ & $(0,21)$ & $(0,75)$ & $(0,17)$ \\
\hline \multirow[t]{2}{*}{50} & $30-45$ & 4,04 & 270,00 & 36,00 & 64,00 & 0,00 & 97,20 & 172,80 & 0,00 \\
\hline & & $(0,55)$ & $(36,87)$ & $(7,22)$ & $(7,22)$ & $(0,00)$ & $(0,33)$ & $(0,49)$ & $(0,00)$ \\
\hline \multirow[t]{2}{*}{50} & $45-60$ & 2,66 & 178,00 & 47,00 & 53,00 & 0,00 & 83,66 & 94,34 & 0,00 \\
\hline & & $(0,29)$ & $(19,38)$ & $(3,77)$ & $(3,77)$ & $(0,00)$ & $(0,21)$ & $(0,13)$ & $(0,00)$ \\
\hline \multirow[t]{2}{*}{50} & $60-75$ & 1,86 & 124,00 & 70,00 & 30,00 & 0,00 & 86,80 & 37,20 & 0,00 \\
\hline & & $(1,00)$ & $(66,34)$ & $(14,6)$ & $(14,6)$ & $(0,00)$ & $(0,49)$ & $(0,55)$ & $(0,00)$ \\
\hline Média & & 3,82 & 255,00 & 45,16 & 42,84 & 12,26 & 88,68 & 128,21 & 66,48 \\
\hline
\end{tabular}

MF (massa de forragem), DF (densidade de forragem), LV (proporção de lâminas foliares verdes), CV (proporção de colmos verdes), MM (proporção de material morto), MLV (massa de lâminas foliares verdes), MCV (massa de colmos verdes), MMM (massa de material morto).

Fonte: Dados da pesquisa. 
Com relação ao estrato 1 , mais rente ao solo, a proteína bruta das lâminas foliares verdes (PBL) e dos colmos verdes (PBC) não sofreram alterações em função da altura do pasto (Quadro 2). Nos estratos 2 e 3 (Quadros 2 e 3), a PBL apresentou uma resposta quadrática crescente aos incrementos na altura $(\mathrm{p}<0,01)$. No estrato 4 , o teor proteico das lâminas aumentou linearmente com a altura (Quadros 3 e 4), o que mostra que quanto maior a altura do pasto, maior o valor desse nutriente nessa camada. Houve uma resposta quadrática de PBC nos estratos 2 e 3 (Quadro 2 e 3), e quadrática no estrato 4 (Quadro 3). No trabalho de Silva et al. (2012), o teor de proteína bruta diminuiu com o aumento da altura da pastagem, que foi manejada nas alturas 10, 20, 30 e $40 \mathrm{~cm}$.

Os teores médios de PB da lâmina, do colmo do material morto foram 11,0; 6,4 e 4,8\%, respectivamente (Quadro 5). A média da PB das lâminas no pasto de $10 \mathrm{~cm}$ foi de $9,85 \%$ e no pasto de $50 \mathrm{~cm}, 9,24 \%$. Esses valores não atendem às exigências para novilhos em engorda segundo o NRC (2000).
A FDNL e FDNC apresentaram incrementos em função da altura do pasto $(\mathrm{p}<0,01)$ para os estratos 3 e 4 . O mesmo padrão foi visto no trabalho de Paula et al. (2012), quando os pastos de capim Marandu foram manejados com 30 e $45 \mathrm{~cm}$. Isto indica que pastagens mais altas apresentam maior lignificação nas camadas verticais da planta, decorrentes do envelhecimento dos componentes morfológicos. O estrato mais rente ao solo, independente da altura utilizada, apresentou lâminas e colmos verdes com maiores teores de FDN (Quadro 5), o que implica em menor digestibilidade da planta (VAN SOEST, 1994). Nos estratos superiores, aqueles mais acessíveis aos animais, foram menores os teores de FDN, corroborando com Paula et al. (2012), que também trabalharam com capim Marandu. Esta constatação é importante, uma vez que existe correlação negativa entre teor de FDN da planta (folhas e colmos) e o consumo animal, limitando a capacidade de consumo (MERTENS, 1994).

Quadro 5 - Médias e desvios padrões dos componentes químicos, nos estratos verticais do capim Marandu, em função de diferentes alturas de pastejo

\begin{tabular}{|c|c|c|c|c|c|c|c|}
\hline Altura & Estratos & PBL & PBC & PBM & FDNL & FDNC & FDNM \\
\hline$(\mathrm{cm})$ & $(\mathrm{cm})$ & $\%$ & $\%$ & $\%$ & $\%$ & $\%$ & $\%$ \\
\hline \multirow[t]{2}{*}{10} & $0-15$ & 10,80 & 7,50 & 7,40 & 51,10 & 65,80 & 66,60 \\
\hline & & $(7,52)$ & $(1,10)$ & $(1,01)$ & $(3,07)$ & $(4,01)$ & $(2,56)$ \\
\hline \multirow[t]{2}{*}{10} & $15-30$ & 8,90 & 7,20 & 2,60 & 49,30 & 61,70 & 0,00 \\
\hline & & $(0,96)$ & $(0,53)$ & $(0,42)$ & $(2,82)$ & $(2,48)$ & $(0,00)$ \\
\hline \multirow[t]{2}{*}{17} & $0-15$ & 10,70 & 5,70 & 5,80 & 52,70 & 65,50 & 67,30 \\
\hline & & $(1,38)$ & $(0,77)$ & $(0,70)$ & $(25,70)$ & $(2,41)$ & $(3,76)$ \\
\hline \multirow[t]{2}{*}{17} & $15-30$ & 11,40 & 7,20 & 4,80 & 51,30 & 63,30 & 60,20 \\
\hline & & $(2,13)$ & $(0,81)$ & $(2,47)$ & $(2,84)$ & $(2,58)$ & $(34,01)$ \\
\hline \multirow[t]{2}{*}{17} & $30-45$ & 10,30 & 5,30 & - & 49,10 & 57,50 & - \\
\hline & & $(5,17)$ & $(4,20)$ & - & $(26,13)$ & $(32,40)$ & - \\
\hline \multirow[t]{2}{*}{18} & $0-15$ & 12,70 & 6,40 & 5,80 & 53,90 & 63,10 & 50,10 \\
\hline & & $(1,57)$ & $(0,37)$ & $(1,24)$ & $(1,97)$ & $(2,10)$ & $(33,46)$ \\
\hline \multirow[t]{2}{*}{18} & $15-30$ & 13,70 & 8,30 & 6,30 & 50,80 & 60,60 & 61,10 \\
\hline & & $(1,49)$ & $(1,48)$ & & $(2,00)$ & $(3,08)$ & $(0,00)$ \\
\hline \multirow[t]{2}{*}{29} & $0-15$ & 10,15 & 6,10 & 5,50 & 53,58 & 64,50 & 65,80 \\
\hline & & $(0,54)$ & $(0,39)$ & $(1,10)$ & $(2,18)$ & $(2,04)$ & $(2,34)$ \\
\hline \multirow[t]{2}{*}{29} & $15-30$ & 11,30 & 8,40 & - & 52,90 & 60,40 & - \\
\hline & & $(0,43)$ & $(0,88)$ & - & $(1,64)$ & $(2,54)$ & - \\
\hline \multirow[t]{2}{*}{29} & $30-45$ & 12,90 & 9,60 & - & 50,10 & 59,50 & - \\
\hline & & $(0,15)$ & $(4,82)$ & - & $(0,91)$ & $(29,85)$ & - \\
\hline \multirow[t]{2}{*}{32} & $0-15$ & 11,30 & 6,90 & 6,50 & 52,40 & 62,20 & 65,10 \\
\hline & & $(0,88)$ & $(0,62)$ & $(1,36)$ & $(2,05)$ & $(1,66)$ & $(3,02)$ \\
\hline \multirow[t]{2}{*}{32} & $15-30$ & 12,40 & 8,10 & - & 50,80 & 60,50 & - \\
\hline & & $(0,45)$ & $(0,66)$ & - & $(1,73)$ & $(1,76)$ & - \\
\hline \multirow[t]{2}{*}{32} & $30-45$ & 13,60 & 6,90 & - & 49,30 & 57,80 & - \\
\hline & & $(1,22)$ & $(1,15)$ & - & $(1,48)$ & $(2,29)$ & - \\
\hline \multirow[t]{2}{*}{32} & $45-60$ & 12,80 & - & - & 47,00 & 0,00 & - \\
\hline & & - & - & - & $(0,00)$ & $(0,00)$ & - \\
\hline \multirow[t]{2}{*}{50} & $0-15$ & 8,80 & 5,40 & 4,72 & 53,30 & 64,90 & 64,50 \\
\hline & & $(1,89)$ & $(3,99)$ & $(1,14)$ & $(0,44)$ & $(2,06)$ & $(1,63)$ \\
\hline \multirow[t]{2}{*}{50} & $15-30$ & 8,40 & 4,90 & 3,80 & 50,30 & 64,80 & 62,10 \\
\hline & & $(2,35)$ & $(3,23)$ & $(1,79)$ & $(5,97)$ & $(1,18)$ & $(0,58)$ \\
\hline \multirow[t]{2}{*}{50} & $30-45$ & 9,10 & 5,70 & - & 54,20 & 62,10 & - \\
\hline & & $(3,62)$ & $(2,57)$ & - & $(3,31)$ & $(2,82)$ & - \\
\hline \multirow[t]{2}{*}{50} & $45-60$ & 10,10 & 6,30 & - & 53,50 & 60,10 & - \\
\hline & & $(3,83)$ & $(2,55)$ & - & $(0,13)$ & $(1,32)$ & - \\
\hline \multirow[t]{2}{*}{50} & $60-75$ & 9,80 & 6,60 & - & 54,20 & 60,50 & - \\
\hline & & $(2,53)$ & $(1,72)$ & - & $(1,07)$ & $(0,46)$ & - \\
\hline Média & & 11,00 & 6,44 & 4,83 & 51,56 & 58,67 & 56,28 \\
\hline
\end{tabular}

PBL (proteína bruta das lâminas foliares verdes), PBC (proteína bruta dos colmos verdes), PBM (proteína bruta do material morto (PBM), FDNL (fibra em detergente neutro das lâminas foliares verdes), FDNC (fibra em detergente neutro dos colmos verdes), FDNM (fibra em detergente neutro do material morto). Fonte: Dados da pesquisa. 
O estrato 1, a densidade de lâminas apresentou aumento quadrático crescente com a altura (Quadro 6), com o pasto de $10 \mathrm{~cm}, 30 \mathrm{~kg} / \mathrm{ha} . \mathrm{cm}$ de MS, e no de $50 \mathrm{~cm}, 63,8 \mathrm{~kg} / \mathrm{ha} . \mathrm{cm}$ de MS. Os valores de densidade dependem, além da altura e manejo utilizados, da genética da planta, como por exemplo se observa em estudos com Brachiaria decumbens cv. Basilisk (SANTOS et al., 2010). Os autores analisaram quatro alturas de manejo $(10,20,30$ e $40 \mathrm{~cm})$ e verificaram que a densidade de lâmina foliar verde diminuiu com o aumento da altura do pasto.

Quadro 6 - Equação de regressão e coeficientes de determinação $\left(\mathrm{R}^{2}\right)$ da densidade dos componentes morfológicos, nos estratos verticais 1 e 2, do capim Marandu em função de diferentes alturas de pastejo

\begin{tabular}{|l|c|c|c|c|}
\hline \multicolumn{1}{|c|}{ Variáveis do } & \multicolumn{2}{c|}{ Estrato 1 (0-15 cm) } & \multicolumn{2}{c|}{ Estrato 2 (15-30 cm) } \\
\hline \multicolumn{1}{|c|}{ Pasto } & Equação de regressão & $\mathrm{R}^{2}$ & Equação de regressão & $\mathrm{R}^{2}$ \\
\hline DEL $(\mathrm{kg} / \mathrm{ha}$.com) & $\mathrm{Y}=6,18+8,40^{\mathrm{a}}-0,15 \mathrm{X}^{2}$ & 0,25 & $3^{*}$ & - \\
\hline DEC $(\mathrm{kg} / \mathrm{ha} . \mathrm{cm})$ & $186,28^{*}$ & & $113,2^{*}$ & - \\
\hline DEM $(\mathrm{kg} / \mathrm{ha} . \mathrm{cm})$ & $\mathrm{Y}=326,02-12,89 \mathrm{X}+0,17 \mathrm{X}^{2}$ & 0,37 & $10,7^{*}$ & - \\
\hline
\end{tabular}

DEL (densidade de lâminas foliares verdes), DEC (densidade de colmos verdes) e DEM (densidade de material morto), ${ }^{*}$ (média), p-valor $>0,05$.

Fonte: dados da pesquisa.

No presente estudo, a densidade de colmos foi superior à de lâminas e de material morto em todas as alturas, exceto no manejo a $10 \mathrm{~cm}$, no qual prevaleceu a densidade de material morto. No estrato 1 , os valores de mínimo e máximo para densidade de colmos foram de $124 \mathrm{~kg} / \mathrm{ha} . \mathrm{cm}$ de MS no pasto de $10 \mathrm{~cm}$ e $249 \mathrm{~kg} / \mathrm{ha} . \mathrm{cm}$ de MS. Já no pasto de $18 \mathrm{~cm}$ obteve uma média de 186,28 kg/ha.cm de MS.

A densidade de material morto apresentou efeito quadrático em função da altura. Em um estudo realizado por Fontes et al. (2003), com Brachiaria brizantha manejada entre 10 e 40 $\mathrm{cm}$, verificou-se que o acúmulo de folhas não sofreu influência das diferentes alturas, que corresponde à proporção constante da parte superior do perfil vertical da planta, independente da altura de manejo pastejável.

No estrato 2, a densidade dos componentes não apresentou variação com a altura ( $p>0,05)$, e as médias para densidade de lâminas, colmos e material morto foram de 85,3; 113,2 e 10,7 $\mathrm{kg} / \mathrm{ha} . \mathrm{cm}$ de MS, respectivamente (Quadro 6).

No estrato 3, ocorreu incremento linear para lâminas e quadrático crescente para colmos (Quadro 7), sem presença de material morto. Houve superioridade da densidade de lâminas em relação a colmos até a altura de $43 \mathrm{~cm}$, ponto em que há incremento na densidade de colmos.

Quadro 7 - Equação de regressão e coeficientes de determinação $\left(\mathrm{R}^{2}\right)$ da densidade dos componentes morfológicos, nos estratos verticais 3 e 4, do capim Marandu em função de diferentes alturas de pastejo

\begin{tabular}{|l|c|c|c|c|}
\hline \multicolumn{1}{|c|}{ Variáveis do } & Estrato 3 $\mathbf{( 3 0 - 4 5} \mathbf{~ c m})$ & $\mathbf{R}^{\mathbf{2}}$ & Estrato 4 (45-60 cm) & $\mathbf{R}^{\mathbf{2}}$ \\
\hline \multicolumn{1}{|c|}{ Pasto } & Equação de regressão & $\mathrm{R}^{2}$ & Equação de regressão & $\mathrm{R}^{2}$ \\
\hline DEL $(\mathrm{kg} / \mathrm{ha} . \mathrm{cm})$ & $\mathrm{Y}=25,25+2,70^{\mathrm{a}}$ & 0,59 & $\mathrm{Y}=31,43-3,61 \mathrm{X}+0,092 \mathrm{X}^{2}$ & 0,90 \\
\hline DEC $(\mathrm{kg} / \mathrm{ha} . \mathrm{cm})$ & $\mathrm{Y}=55,75-6,05 \mathrm{X}+0,16 \mathrm{X}^{2}$ & 0,85 & $\mathrm{Y}=43,75-4,84 \mathrm{X}+0,11 \mathrm{X}^{2}$ & 0,94 \\
\hline
\end{tabular}

DEL (densidade de lâminas foliares verdes), DEC (densidade de colmos verdes), p-valor $>0,05$.

Fonte: Dados da pesquisa.

A pastagem que foi mantida em $50 \mathrm{~cm}$ apresentou densidade de colmos superior a de lâminas em todos os estratos do perfil vertical da planta. Aplicando a equação do Quadro 7, também para o estrato 3, a densidade de lâminas foi maior do que à de colmo. No estrato 4 , ocorreu um equilíbrio entre os dois componentes apresentando densidades reais de $90 \mathrm{~kg} / \mathrm{ha} . \mathrm{cm}$ de MS para lâminas e $94 \mathrm{~kg} / \mathrm{ha} . \mathrm{cm}$ de MS para colmos. O estrato 4 obteve comportamento quadrático decrescente, apresentando maiores valores nos pastos mais altos, sem presença de material morto.

\section{Conclusão}

O manejo em diferentes alturas na Urochloa brizantha cv. Marandu altera as características dos estratos verticais do pasto, principalmente, nos estratos acima de $15 \mathrm{~cm}$. O estrato superior $(45-60 \mathrm{~cm})$ apresenta maior densidade de lâminas foliares verdes e o estrato próximo ao solo apresenta menor sensibilidade às variações na estrutura da planta.

\section{Referências}

AOAC - Association of Official Analytical Chemistis. Official methods of analysis. Washington: AOAC, 1990.

BAUER, M. O. et al. Produção e características estruturais de cinco forrageiras do gênero Brachiaria sob intensidades de cortes intermitentes. Ciênc. Anim. Bras., v.12, n.1, p.17-25, 2011. doi: 10.5216/cab.v12i1.4817

FONTES, J.G.G. et al. Acúmulo de massa seca em cultivares de Brachiaria brizantha submetida a intensidades de desfolhação. Semina: Ciênc. Agrárias, v.35, n.3, p.1425-1438, 2014. doi: 10.5433/1679-0359.2014v35n3p1425

GONÇALVES, A.C. Características morfogênicas e padrões de desfolhação em pastos de capim Marandu submetidos a regimes de lotação contínua. Piracicaba: Universidade de São Paulo, 2002.

HOLDERBAUN, J.F.; SOLLENBERG, K.H. Canopy structure and nutritive value of limpograss pastures during mid-summer to early autumn. Agronomy J., v.84, p.11-16, 1992.

IWAMOTO, B. S. et al. Características morfogênicas do CapimTanzânia fertilizado ou não com nitrogênio nas estações do ano. 
Bioscience Journal, v.31, n.1, p.181-193, 2015.

MERTENS, D.R. Regulation of forage intake. In: FAHEY JR., G.C. Forage quality evaluation and utilization. American Society of Agronomy. Madison, 1994. p.450-493.

NATIONAL RESEARCH COUNCIL - NRC. Nutritional requirements of beef cattle.

PAULA, C. C. L. et al. Estrutura do dossel, consumo e desempenho animal em pastos de capim Marandu sob lotação contínua. Arq. Bras. Med. Vet. Zootec., v.64, n.1, p.169-176, 2012. doi: 10.1590/ S0102-09352012000100024.

SANTOS, M. E. R. et al. Estrutura do pasto de capim Braquiária com variação de alturas. Rev. Bras. Zootec., v.39, n.10, p.21252131, 2010.

SANTOS, M. E. R. et al. O manejo da desfolhação utilizado no inverno modifica a estrutura vertical do capim-marandu no verão subsequente. Enciclop. Biosfera, v.11, n.21, p.3526-3534, 2015.

SILVA, S. C. et al. Grazing behaviour, herbage intake and animal performance of beef cattle heifers on Marandu palisade grass subjected to intensities of continuous stocking management. $J$. Agricul. Scie., v.151, p.727-739, 2012.

SIBCS - Sistema Brasileiro de Classificação de Solos. EMBRAPA, 2013.

VAN SOEST, P. J. Nutritional ecology of the ruminant. Ithaca: Cornell University Press, 1994.

VAN SOEST, P.J; ROBERTSON, J.B.; LEWIS, B.A. Methods for dietary fiber, neutral detergent fiber, and nonstarch polysaccharides in relation to animal nutrition. J. Dairy Scie., v.74, n.10, p.35833597, 1991. 